\title{
Manifestações orais em pacientes imunodeprimidos pelo Vírus da Imunodeficiência Humana (HIV): revisão da literatura
}

\author{
Oral manifestations in patients immunified by Human Immunodeficiency Virus (HIV): \\ literature revision
}
Manifestaciones orales en pacientes inmunificados por Virus de Inmunodeficiencia Humana $(\mathrm{VIH})$ : revisión de literatura

\begin{abstract}
Orlando de Jesus Londono Dominguez Filho', Edinelza Costa Viana1, Willian Guimarães Pessoa ${ }^{1}$, Pedro Rauel Cândido Domingos ${ }^{1 *}$.
\end{abstract}

\section{RESUMO}

Objetivo: Descrever as manifestações orais em pacientes imunodeprimidos portadores do HIV. Métodos: Trata-se de uma Revisão de Literatura onde as buscas dos artigos científicos foram realizadas nas bases de dados Scientific Electronic Library Online (SciELO) e Literatura Latino-Americana em Ciências da Saúde (LILACS), cujo critério de inclusão foi, os artigos científicos realizados no Brasil, em língua portuguesa, que foram publicados no período de 2009 a 2020. Resultados: Nesta revisão da literatura foi possível evidenciar que as principais lesões orais relacionadas ao HIV constituem a candidíase oral, leucoplasia pilosa, sarcoma de Kaposi e Gengivite ulcerativa necrosante associada ao HIV, além de outras ulcerações recorrentes. Este estudo demonstra de forma detalhada as formas de tratamento para cada manifestação oral citada anteriormente. Considerações finais: A infecção pelo vírus da imunodeficiência humana (HIV) é uma pandemia caracterizada por alteração no sistema imunológico onde se observa uma progressiva depleção dos linfócitos $\mathrm{T} \mathrm{CD}^{+}$, o que propicia o aparecimento de infecções oportunistas, onde as manifestações orais provêm do surgimento dessas infecções e podem sinalizar uma imunossupressão nos indivíduos infectados pelo HIV.

Palavras-chave: AIDS, Manifestações orais, Odontologia.

\begin{abstract}
Objective: To describe oral manifestations in immunocompromised patients with HIV. Methods: This is a Literature Review where searches for scientific articles were carried out in the Scientific Electronic Library Online (SciELO) and Latin American Literature in Health Sciences (LILACS) databases, whose inclusion criterion was scientific articles performed in Brazil, in Portuguese, which were published from 2009 to 2020. Results: In this literature review, it was possible to show that the main oral lesions related to HIV constitute oral candidiasis, hairy leukoplakia, Kaposi's sarcoma and necrotizing ulcerative gingivitis associated with HIV, in addition to other recurrent ulcerations. This study demonstrates in detail the forms of treatment for each oral manifestation mentioned above. Final considerations: Infection by the human immunodeficiency virus (HIV) is a pandemic characterized by changes in the immune system where progressive depletion of CD4+ $\mathrm{T}$ lymphocytes is observed, which promotes the appearance of opportunistic infections, where oral manifestations come from the appearance of these infections and may signal immunosuppression in HIVinfected individuals.
\end{abstract}

Key words: AIDS, Oral manifestations, Dentistry.

${ }^{1}$ Centro Universitário do Norte (UNINORTE), Manaus - AM. *E-mail: pedrorauel@gmail.com 


\section{RESUMEN}

Objetivo: Describir las manifestaciones bucales en pacientes inmunodeprimidos con VIH. Métodos: Se trata de una Revisión de Literatura donde se realizaron búsquedas de artículos científicos en las bases de datos de la Biblioteca Electrónica Científica en Línea (SciELO) y Literatura Latinoamericana en Ciencias de la Salud (LILACS), cuyo criterio de inclusión fueron artículos científicos. realizado en Brasil, en portugués, que fueron publicados de 2009 a 2020. Resultados: En esta revisión de la literatura se pudo demostrar que las principales lesiones orales relacionadas con el VIH son la candidiasis oral, la leucoplasia pilosa, el sarcoma de Kaposi y la gingivitis ulcerosa necrosante asociada al $\mathrm{VIH}$, además de otras ulceraciones recurrentes. Este estudio demuestra en detalle las formas de tratamiento para cada manifestación oral mencionada anteriormente. Consideraciones finales: La infección por el virus de la inmunodeficiencia humana (VIH) es una pandemia caracterizada por cambios en el sistema inmunológico donde se observa un agotamiento progresivo de los linfocitos T CD4, lo que promueve la aparición de infecciones oportunistas, donde las manifestaciones orales provienen de la aparición de estas infecciones. y puede indicar inmunosupresión en individuos infectados por el VIH.

Palabras clave: AIDS, Manifestaciones orales, Odontología.

\section{INTRODUÇÃO}

A Síndrome da Imunodeficiência Adquirida (SIDA - AIDS em inglês) é uma doença infecciosa de natureza viral caracteriza-se pela depressão do sistema imunológico, o que possibilita o desenvolvimento de diversas infecções oportunistas. O agente etiológico responsável pela enfermidade é o Vírus da Imunodeficiência Humana (HIV em inglês), agente viral pertencente ao gênero Lentivirus, da família Retroviridae, sendo responsável por grande morbimortalidade (MORAES DCA, et al., 2014).

De acordo com o Ministério da Saúde (2019) no Brasil, no ano de 2018, 43.941 novos casos de HIV e 37.161 casos de AIDS, foram diagnosticados, onde os mesmos foram notificados no Sinan, o que constitui uma taxa de detecção de 17,8/100.000 habitantes (2018), totalizando, no período de 1980 a junho de 2019, 966.058 casos de AIDS detectados no país.

Dados de estimativas mundiais apontam que mais de sete mil pessoas são infectadas com o vírus diariamente e que a cada 20 segundos uma pessoa morre em consequência de alguma doença relacionada a infecção pelo HIV. A AIDS, patologia associada a essa infecção, é considerada a $5^{\mathrm{a}}$ causa mais comum de morte entre adultos, onde se observa que as mulheres são as mais afetadas, compreendendo a idade de 15 e 49 anos. No Brasil estima-se que 718 mil pessoas são portadoras de HIV, dessas apenas 686.478 foram notificadas, sendo que anualmente surgem 37.446 novos casos, onde 13.781 foram descritos como novos somente no primeiro semestre de 2013 (CARVALHO RC e HAMER ER, 2017).

Patologias orais afetam cerca de $60 \%$ dos indivíduos com HIV e $80 \%$ daqueles que já manifestação a AIDS, os quais são importantes determinantes para o diagnóstico da infecção, visto que são consideradas oportunistas, geralmente não ocorrendo naqueles imunocompetentes. Além disso, as alterações bucais demonstram o quadro de debilidade imunológica dos indivíduos infectados pelo HIV, visto que a uma relação direta entre esta depressão imunológica e a ocorrência das manifestações clínicas. A depleção dos linfócitos $\mathrm{T} \mathrm{CD}^{+}$, a elevada carga viral, comportamentos de higiene bucal inadequadas, assim como o tabagismo, tem sido importantes fatores associados ao surgimento e agravamento precoce das alterações bucais em pacientes com AIDS (GASPARIN AB, et al., 2009).

As manifestações orais podem ter etiologias diferentes, sendo causadas por bactérias, fungos, vírus ou, ainda, de natureza neoplásica. Essas lesões são divididas em três grupos com base nas suas características clínicas: Grupo 1 - lesões associadas à infecção pelo HIV, como candidose bucal, leucoplasia pilosa, Sarcoma de Kaposi, eritema gengival linear, gengivite ulcerativa necrosante, periodontite ulcerativa necrosante, e linfoma não Hodgkin; Grupo 2 - inclui úlceras atípicas, doenças das glândulas salivares e infecções virais, como Citomegalovirose (infecção por Citomegalovírus), herpes simples, papiloma vírus e Herpes Zoster; Grupo 3 - apresenta as lesões mais raras, como osteomielite difusa e carcinoma de células escamosas (MOTTA WKS, et al., 2014). 
Nesse contexto, o objetivo deste trabalho foi descrever as principais manifestações orais em pacientes imunodeprimidos portadores do HIV, assim como as causas e tratamentos relacionados.

\section{REVISÃO BIBLIOGRÁFICA}

A Síndrome da Imunodeficiência Adquirida (ing.: Acquired Immuno Deficiency Syndrome - AIDS) é considerada um grave problema de saúde pública a décadas, sendo objeto de estudo desde a de 1980 com os registros dos primeiros casos, os quais aumentaram progressivamente nos anos seguintes, afetando muitas pessoas por todo o mundo. A transmissão heterossexual passou a ser a principal via de transmissão, a qual vem apresentando maior tendência de crescimento quando comparada a relação homossexual. Acompanhada desse comportamento, a infeção feminina vem ganhando maior expressividade nos últimos anos em diversos países, havendo também um aumento da transmissão materno-infantil em decorrência dessa maior participação feminina. Nota-se, ainda, um processo de interiorização dos casos, acompanhando o crescimento de pequenos municípios (menos de 50 mil habitantes), além de ser mais prevalente naqueles de menor escolaridade (DANTAS CC, et al., 2017).

O Vírus da Imunodeficiência Humana (ing.: Human Immunodeficiency Viruses - HIV) é o agente etiológico da AIDS e é, essencialmente, transmitido a partir relações sexuais desprotegidas, contudo outras formas de transmissão são conhecidas, como o compartilhamento de agulhas e seringas, a gravidez, o parto e a amamentação. A ocorrência da AIDS, patologia relacionada a uma diversidade de manifestações clínicas decorrentes da incompetência do sistema imunológico frente a infecções oportunistas, é desencadeada a partir da perda gradual de leucócitos, especialmente os linfócitos T CD4+, após a infeção pelo HIV, o que leva a uma ineficiência progressiva, quando não tratada, da defesa humoral e celular do organismo. Como resultado dessa infecção, a susceptibilidade do organismo humano a infecções e doenças oportunistas, possibilitadas pela fragilidade do organismo, é aumentada. Protocolos terapêuticos atuais para infecção pelo HIV possibilitam a redução da carga viral e dos efeitos depressivos do sistema imunológico, mas ainda não são suficientes para cura (CASTRO MC e SILVA MA, 2013).

A infecção pelo HIV e a sua evolução para a AIDS, que inclui uma diversidade de manifestações clínicas decorrentes da debilidade imunológica provocada pela infecção, é um problema que afeta uma diversidade de países no mundo e seu controle é o objetivo de todos eles, havendo campanhas internacionais voltadas a essa temática. Apesar de ser uma infecção incurável na atualidade, a partir da terapêutica convencional, o uso de antirretrovirais possibilitou a um curso crônico para essa infecção, trazendo uma maior expectativa de vida aos acometidos por ela. Apesar disso a infecção, mesmo sem a evolução para a AIDS, apresenta algumas limitações a vida dos soropositivos. Por esse motivo abordagens que visem intensificar medidas de prevenção efetivas da infecção são fundamentais para diminuir a sua propagação e os impactos dessa infecção na vida de outas pessoas (RODRIGUES FRA, et al., 2015).

O controle terapêutico, através do uso dos antiretrovirais, mantém a carga viral baixa e linfócitos T CD4+ em quantidade suficiente, e podem ser relacionados a redução da ocorrência de lesões bucais, pois a resposta imunológica é mantida, levando a uma menor susceptibilidade a infecções oportunistas e a sua não ocorrência (HARTMANN A, et al., 2016).

Atualmente, o perfil da HIV/AIDS tem se modificado, uma vez que estudos têm mostrado um crescimento do número de casos entre mulheres, heterossexuais, pessoas entre 15 e 24 anos, adultos acima de 50 anos, indivíduos com baixo grau de instrução, como indicador socioeconômico baixo e em populações de regiões menos urbanizadas (SILVA RAR, et al., 2016).

No mundo, a região da África subsaariana continua sendo a mais atingida, com cerca de $60 \%$ das pessoas vivendo com HIV, onde mulheres representam $58 \%$ deste total, mas áreas como o Caribe, o Leste europeu e a Ásia central, as quais apresentam prevalência de cerca de $1 \%$ na população em geral, são áreas relacionadas a um cenário epidêmico (MARTINS TA, et al., 2014). Esse caráter epidêmico também vem sendo desenhando no Brasil, o qual apresenta elevação observada nos últimos anos nas incidências de HIVe AIDS em algumas regiões do país (CABRAL JVB, et al., 2015). 
No Brasil, os primeiros casos de AIDS, notificados, aconteceram em 1982. A ocorrência de pacientes diagnosticados com este agravo, nesse período, estava restrita ao eixo Rio - São Paulo, sugerindo ter sido o Sudeste o foco inicial da disseminação dessa epidemia no Brasil (ABREU SR, et al., 2016). No país, considerando o período de 1980 até junho de 2019, já foram notificados 966.058 casos de AIDS e 338.905 óbitos relacionados a infecção pelo HIV. A média de casos anual na última década foi de 17,8 casos/100 mil hab. em 2018, segundo dados oficiais do Ministério da Saúde (2019). As maiores taxas de detecção de AIDS em 2018 foram registradas nas regiões Norte e Sul, com 25,1 e 22,8 casos/100 mil hab., mas com tendência de crescimento para região Norte e de decrescimento para a Sul. Outra região que apresentou tendência de crescimento nos últimos anos foi a Nordeste, com taxa de detecção de 15,8 casos de AIDS / 100 mil hab. em 2018 (BRASIL, 2019).

Rwenyonyi CM, et al. (2011) realizaram estudo transversal no Ambulatório de Doenças Infecciosas Pediátricas (PIDC) do Hospital Mulago, sendo identificada a prevalência de manifestações orais em crianças infectadas pelo HIV de $73 \%$, com variação entre as prevalências de pacientes em tratamento e sem tratamento de 67,8 a $78,2 \%$, respectivamente, não sendo verificado grandes variações entre as faixas etárias padronizadas nem entre os sexos dos afetados. Na revisão da literatura realizada nesse estudo, foi apontado que a variação na prevalência de manifestações orais no continente Africano varia de 1,5 a $94 \%$, que prevalências de até $72 \%$ podem ser vistas em países considerados desenvolvidos e que no Brasil podem ser de até $61 \%$.

Segundo trabalho realizado por Chagas MV, et al. (2009), com 100 pacientes com HIV/AIDS no Amazonas/Brasil, as manifestações bucais observadas foram mais frequentes entre os 17 e 68 anos, com média de idade dos afetados do sexo masculino de 36,78 anos e do feminino de 32,36 anos, havendo um notável predomínio das manifestações no sexo masculino (70\% dos afetados) em relação ao feminino. Dentre as diferentes alterações observadas, as mais prevalentes foram a candidíase (54\%) em diferentes formas (pseudomembranosa, eritematosa e queilite angular), a gengivite $(25 \%)$ e a periodontite (18\%).

Pakfetrat A, et al. (2015), em estudo transversal com pacientes portadores de HIV/AIDS os quais foram submetidos a exame oral durante um período de 15 meses de 2008 a 2010, destacaram que a idade média dos pacientes afetados era de $36,2( \pm 8,1$ anos) e que a prevalência no sexo masculino era expressivamente maior naquele grupo $(82,7 \%)$, não havendo diferença entre as medias de idade observadas entre os homens (36,6 $\pm 7,9$ anos) e a média geral. As mulheres observadas apresentavam idade média um pouco inferior, mas sem diferença significativa $(34,1 \pm 9,1$ anos). Os achados mais comuns da mucosa oral, em ambos os sexos, foram periodontite grave (30\%), candidíase pseudomembranosa (26\%) e língua pilosa (26\%).

Davoodi P, et al. (2010), ao analisarem 100 pacientes HIV positivos em um estudo transversal, selecionados aleatoriamente entre os participantes do Centro de Consulta Comportamental de Kermanshah, no Irã, observaram as seguintes manifestações clinicas no grupo analisado: cárie galopante (54), doença periodontal (44), hiperpigmentação (42), Candidíase eritematosa (36), xerostomia (20), queilite angular (17), leucoplasia (16), língua peluda (14), aumento de glândulas salivares (11), candidíase pseudomembranosa (7) e eritema gengival linear (6). Dentre as manifestações com menor ocorrência foram destacadas a leucoplasia pilosa (4), líquen plano da língua (4), línguas fissuradas (3), periodontite ulcerativa necrosante (2), verrugas orais (2), fibromas de irritação (2) e papiloma (1), evidenciando a diversidade de manifestações orais que podem ser observadas nesses pacientes.

Segundo estudo realizado por Paulique NC, et al. (2017), a candidíase, doença provocada a partir da infecção por fungos Candida albicans, pode ser caracterizada em quatro tipos de manifestações clínicas: Pseudomembranosa; Eritematosa; Hiperplásica; e Queilite Angular. Trata-se de uma alteração oral comumente encontrada nos indivíduos portadores de HIV ou que desenvolveram a AIDS, a qual é deflagrada pelo estado de diminuição da imunidade do organismo. Dentre os tipos de manifestações apontados, a Candidíase Pseudomembranosa é a forma mais encontrada nesses pacientes, sendo caracterizada pela presença de pseudomembranas esbranquiçadas ou amareladas, as quais são facilmente removidas utilizando procedimentos mecânicos com a raspagem.

A leucoplasia pilosa oral (LPO) caracteriza-se por uma lesão composta por uma placa branca na mucosa, sendo assinalada, histopatologicamente, por um padrão de certa forma característico de hiperceratose e 
hiperplasia epitelial. Muitos casos de LPO ocorrem na margem lateral da língua e seu aspecto pode variar de estrias brancas verticais tênues a áreas leucoplásicas espessas e corrugadas, apresentando uma superfície ceratótica áspera (NEVILLE BW, et al., 2009).

O sarcoma de Kaposi (KS) é caracterizado como uma neoplasia multifocal, originado nas células endoteliais vasculares. Nos Estados Unidos surgiram em associação à infecção pelo HIV, sendo que $15 \%$ a $20 \%$ dos pacientes com AIDS apresenta o SK. Observa-se dentro do tumor presença do herpesvírus humano tipo 8 (HHV-8, o que pode estar associado ao surgimento da neoplasia (NEVILLE BW, et al., 2009).

A gengivite associada ao HIV é caracterizada por uma faixa linear de eritema que envolve a gengiva marginal livre e se estende 2 a $3 \mathrm{~mm}$ apicalmente. A mucosa alveolar e a gengiva apresentam eritema difuso ou puntiforme em vários casos. Esse diagnóstico está atribuído para gengivites que não respondem ao controle rígido de placa e exibem muito eritema, maior do que o esperado para a quantidade de placa no local (NEVILLE BW, et al., 2009).

Ocorrendo com maior frequência e de progressão rápida, entre indivíduos HIV-positivos, a periodontite ulcerativa necrosante, caracteriza-se como necrose gengival, destruição periodontal rápida e perda de osso interproximal, podendo ser descrita como uma progressão da gengivite ulcerativa necrosante em que ocorre perda óssea e perda de inserção periodontal. As lesões geralmente são localizadas, embora possam apresentar-se de forma generalizada após uma depleção expressiva nos linfócitos T CD4+. O tratamento com terapia antibiótica deve ser usado cautelosamente nesses pacientes, para evitar uma candidíase localizada, oportunista e potencialmente séria (BARROS AVM, et al., 2017).

A gengivite ulcerativa necrosante é uma infecção oral bacteriana caracterizada pela inflamação que pode envolver a gengiva livre, inserida e as mucosas vestibulares e jugal. Severo edema, eritema, sangramento espontâneo, com pseudomembrana e necrose. Odor fétido, dor, destruição da papila interdental e evolução rápida. $O$ eritema gengival linear também de origem bacteriana apresenta-se como uma banda eritematosa e edemaciada, na gengiva marginal, a 2 ou $3 \mathrm{~mm}$ de distância da gengiva livre, podendo-se estender-se até a gengiva inserida e mucosa alveolar (FELIPE LCS, et al., 2016).

A estomatite necrosante ocorre nos pacientes que apresentam necrose gengival, caracterizado pelo processo que se prolonga às cristas alveolares e forma áreas de destruição tecidual maciça, o que compromete os tecidos moles ou ainda pode se estender para o osso subjacente, ocasionando grandes sequestros ósseos (NEVILLE BW, et al., 2009).

O estudo realizado por Araújo R, et al. (2018) destaca que a manifestação oral mais comum na infecção pelo HIV é causada, geralmente, pela Candida albicans, promovendo a denominada candidíase. O tratamento nesses casos é realizado com antifúngicos tópicos e sistêmicos, onde bochechos com Nistatina (100.000 unidades $/ \mathrm{ml}$ ) em conjunto com drogas sistêmicas, como o Fluconazol $150 \mathrm{mg}$ e o Itraconazol 100mg, são comumente utilizados. Porém nos pacientes com deficiência imunológica grave, foi observado resistência ao tratamento, sendo necessário associar o Fluconazol com Terbinafina.

Bajpai S e Pazare AR (2010) complementam que a candidíase oral pode se estender a partir da boca e comprometer a faringe, laringe e esôfago também. O tratamento da candidíase oral depende do tipo clínico, distribuição e gravidade da infecção. O tratamento tópico é eficaz para lesões limitadas e acessíveis. Os trociscos de clotrimazol, pastilhas de nistatina e suspensão oral de nistatina são eficazes para candidíase eritematosa e pseudomembranosa leve a moderada. No entanto, o uso prolongado desses agentes pode resultar em cárie dentária significativa devido aos substratos de carboidratos fermentáveis presentes nas formulações. O aumento do risco de cárie pode ser evitado com o uso de uma suspensão oral de nistatina ( 100.000 unidades $/ 5 \mathrm{ml}$, enxágue da boca e expectoração 3 vezes/dia). Os enxágues orais com clorexidina $0,12 \%$ não contêm substrato cariogênico e podem ser igualmente eficazes.

Ao analisar o Protocolo Clínico e Diretrizes Terapêuticas para Manejo da Infecção pelo HIV em Adultos, nota-se que a leucoplasia pilosa caracteriza-se como uma lesão branca, não removível através da raspagem, encontrada geralmente na borda lateral da língua, podendo ainda ser uni ou bilateral. $O$ tratamento é realizado de forma tópica com solução de retinóide à $0,05 \%$, apresentando uma melhora temporária. O tratamento com Zidovudina ${ }^{\circledR}$ para o HIV pode levar a uma significativa regressão (BRASIL, 2018a). 
Hirata CHW (2015) destacou que o Sarcoma de Kaposi, lesões com características neoplásicas que podem afetar diferentes tecidos e órgãos, são encontradas com certa frequência em portadores do HIV. Esse sarcoma apresenta maior frequência de manifestações orais relacionada ao palato mole, o qual pode apresentar máculas vermelho-azuis ou roxo-azuis, além da possiblidade de ocorrência de nódulos. Esses danos destacados apresentam-se inicialmente de maneira assintomática, mas com a evolução de problemas secundários podem ocasionar dificuldades na fala e na mastigação. Assim como em cânceres com outras etiologias, a quimioterapia e a radioterapia podem ser aplicadas no tratamento para o Sarcoma de Kaposi, estando a escolha da abordagem terapêutica a ser utilizada relacionada ao estágio e localização dos tumores.

De acordo com Neville BW, et al. (2009), a gengivite associada ao HIV ocorre devido a uma resposta imune do hospedeiro anormal à bactéria subgengival. Dados da literatura ainda sugerem que esse padrão de gengivite constitui um padrão incomum de candidíase, onde a mucosa alveolar e a gengiva podem apresentar eritema difuso ou puntiforme em uma porcentagem significante dos casos. O tratamento indicado nesta situação são medicamentos antifúngicos sistêmicos como o fluconazol e o cetoconazol.

Contribuindo com o estudo sobre o tratamento odontológico de pessoas com HIV/AIDS, Brasil (2018b), relata que a doença periodontal engloba a gengivite ulcerativa necrosante e a periodontite ulcerativa necrosante, ambas desencadeadas por alterações imunitárias, agravadas por estresse ou má nutrição. $O$ tratamento para ambas é caracterizado por debridamento de tecidos moles e duros necróticos, bochechos com digluconato de clorexidina $0,12 \%$, além de acompanhamento e manutenção à longo prazo. A antibióticoterapia tem sido indicada em pacientes imunossuprimidos, e em casos mais exacerbados da doença. O Metronidazol tem sido o medicamento sistêmico de primeira escolha, em dosagens de $250 \mathrm{mg}$ a $500 \mathrm{mg}$ a cada oito horas por sete dias.

A estomatite necrosante é descrita por Neville BW, et al. (2009), como o processo que pode envolver predominantemente os tecidos moles ou se estender para o osso subjacente, resultando em grandes sequestros ósseos. A estomatite necrosante tem surgido na mucosa oral separada da gengiva (que não recobre osso) onde a perda da inserção periodontal pode ser corrigida através da remoção pelo profissional da placa supra e subgengival, a qual melhora as técnicas de higiene oral pessoal. Os antibióticos de primeira escolha na estomatite necrosante são a penicilina e o metronidazol.

Araújo R, et al. (2018), em seu estudo relataram que conforme é preconizado para todo novo paciente, e de forma que o cirurgião-dentista atue com segurança, é necessário a realização de uma anamnese detalhada, a avaliação do histórico médico, bem como exame completo de cabeça e pescoço, pois o exame clínico e o histórico do paciente podem indicar que o paciente tem HIV/AIDS.

Qualquer paciente deve ser tratado como potencialmente infectado, como uma medida cautelar, em decorrência da impossibilidade de se diferenciar pacientes infectados assintomáticos de pacientes não infectados. Pacientes com alto risco para o HIV, ou aqueles em que a infecção já foi diagnosticada, devem ser tratados da mesma forma que qualquer outro paciente, seguindo todas as medidas de biossegurança universais (DE ARAÚJO R, et al., 2018).

\section{CONSIDERAÇÕES FINAIS}

Apesar de ser conhecida desde a década de 1980, quando os primeiros casos foram descritos, dos diversos trabalhos de pesquisa clínicas e epidemiológicas e dos diversos programas e campanhas de prevenção, a infecção pelo HIV ainda é um grave problema no mundo e afeta muitas pessoas, que muitas vezes desconhecem sua situação sorológica. Manifestações orais observadas pelos profissionais da odontologia, após exame clínico, podem ser indicativos da evolução dessa infecção, auxiliando no diagnóstico da AIDS. A presença de lesões na mucosa oral, apesar da sua grande diversidade e da gravidade variada, origina-se, essencialmente, de infecções oportunistas e são importantes marcadores do estado de imunossupressão dos indivíduos infectados pelo HIV. Conhecer as principais manifestações orais, além de auxiliar no diagnóstico e acompanhamento desses pacientes, fornece informações valiosas aos profissionais dentistas para a decisão dos melhores protocolos terapêuticos e de acompanhamentos desses indivíduos. 


\section{REFERÊNCIAS}

1. ABREU SR, et al. Estudo epidemiológico de pacientes com infecção pelo vírus da imunodeficiência humana/síndrome da imunodeficiência adquirida (HIV/AIDS), Caxias-MA. R. Interd., 2016; 9(4): 132-141.

2. BAJPAI S, PAZARE AR. Oral manifestations of HIV. Contemp Clin Dent, 2010; 1(1): 1-5.

3. BARROS AVM, et al. Doenças periodontais em pacientes HIV positivos: uma revisão da literatura. Braz J Periodontol, 2017; 27(2): 54-60.

4. BRASIL. Ministério da Saúde. Secretaria de Atenção à Saúde. Departamento de Atenção Básica. A saúde bucal no Sistema Único de Saúde. 2018b. <http://bvsms.saude.gov.br/bvs/publicacoes/saude_bucal_sistema_unico_saude.pdf>. Acesso: 23 nov. 2020.

5. BRASIL. Ministério da Saúde. Secretaria de Vigilância em Saúde. Departamento de Doenças de Condições Crônicas e Infecções Sexualmente Transmissíveis - DCCI. Boletim Epidemiológico de HIV e Aids. 2019. Disponível em: <http://www.aids.gov.br/pt-br/pub/2019/boletim-epidemiologico-de-hivaids-2019>. Acesso: 23 nov. 2020.

6. BRASIL. Ministério da Saúde. Secretaria de Vigilância em Saúde. Departamento de Vigilância, Prevenção e Controle das Infecções Sexualmente Transmissíveis, do HIV/Aids e das Hepatites Virais. Protocolo Clínico e Diretrizes Terapêuticas para Manejo da Infecção pelo HIV em Adultos. 2018a. Disponível em: < http://www.aids.gov.br/ptbr/pub/2013/protocolo-clinico-e-diretrizes-terapeuticas-para-manejo-da-infeccao-pelo-hiv-em-adultos >. Acesso: 23 nov. 2020

7. CABRAL JVB, et al. Perfil sociodemográfico, epidemiológico e clínico dos casos de HIV/AIDS em adolescentes no estado de Pernambuco. Revista Uniara, 2015; 18(1): 149-163.

8. CARVALHO RC, HAMER ER. Perfil de alterações no hemograma de pacientes HIV+. RBAC, 2017; 49(1): 57-64.

9. CASTRO MC, SILVA MA. O comportamento dos adolescentes frente ao risco de contaminação com HIV/AIDS. Estudos, 2013; 40(4): 395-418.

10. CHAGAS MV, et al. Manifestações Bucais de Pacientes HIV atendidos na Fundação de Medicina Tropical do Amazonas (FMT-AM). Rev. Fac. Odontol., 2009; 50(3): 10-13.

11. DANTAS CC, et al. Perfil epidemiológico dos pacientes com HIV atendidos em um centro de saúde da região litorânea do estado de rio de janeiro, Brasil, 2010-2011. Arq. Catarin Med., 2017; 46(1): 22-32.

12. DAVOODI P, et al. Oral Manifestations Related To CD4 Lymphocyte Count in HIV-Positive Patients. J Dent Res Dent Clin Dent Prospects, 2010; 4(4): 115-119.

13. ARAÚJO R, et al. Atendimento odontológico aos pacientes com HIV/AIDS. Revista de Odontologia Contemporânea, 2018; 2(1): 28-36.

14. FELIPE LCS, et al. Pacientes com HIV/AIDS na Odontologia e suas Manifestações Bucais. J Orofac Invest, 2016; 3(1): 53-62.

15. GASPARIN AB, et al. Prevalência e fatores associados às manifestações bucais em pacientes HIV positivos atendidos em cidade sul-brasileira. Cad. Saúde Pública, 2009; 25(6): 1307-1315.

16. HARTMANN A, et al. Incidência de Candida spp. na mucosa oral de pacientes infectados pelo Vírus da Imunodeficiência Humana (HIV) no município de Santo Ângelo-RS. R Epidemiol Control Infec, 2016; 6(3): $125-130$.

17. HIRATA CHW. Oral manifestations in AIDS. Braz J Otorhinolaryngol, 2015; 81(2): 120-123.

18. MARTINS TA, et al. Cenário Epidemiológico da Infecção pelo HIV e AIDS no Mundo. Rev Fisioter S Fun., 2014; 3(1): 4-7.

19. MORAES DCA, et al. Adesão de homens vivendo com HIV/Aids ao tratamento antirretroviral. Esc Anna Nery, 2014; 18(4): 676-681.

20. MOTTA WKS, et al. Aspectos demográficos e manifestações clínicas bucais de pacientes soropositivos para 0 HIV/Aids. Rev Odontol UNESP, 2014; 43(1): 61-67.

21. NEVILLE BW, et al. Patologia oral \& maxilofacial. [tradução Danielle Resende Camisasca Barroso... et al.]. Rio de Janeiro: Elsevier, 2009.

22. PAKFETRAT A, et al. Manifestações orais de pacientes infectados com o vírus da imunodeficiência humana. Iran J Otorhinolaryngol, 2015; 27(78): 43-54.

23. PAULIQUE NC, et al. Manifestações bucais de pacientes soropositivos para HIV/AIDS. Arch Health Invest, 2017; 6(6): 240-244.

24. RODRIGUES FRA, et al. Confidencialidade do diagnóstico de HIV: relação entre biopoder e bioética. Almanaque multidisciplinar de pesquisa, 2015; 1(1): 170-184.

25. RWENYONYI CM, et al. Oral Manifestations in HIV/AIDS-Infected Children. Eur J Dent., 2011; 5(3): $291-298$.

26. SILVA RAR, et al. Perfil clínico-epidemiológico de adultos HIV-positivo atendidos em um hospital de Natal/RN. J. res.: fundam. care. online, 2016; 8(3): 4689-4696. 